\title{
The physics program of the CBM experiment
}

\author{
Christian Simon, Ingo Deppner*, and Norbert Herrmann (for the CBM collaboration) \\ Universität Heidelberg \\ Physikalisches Institut, Im Neuenheimer Feld 226, 69120 Heidelberg, Germany \\ E-mail: deppnerephysi.uni-heidelberg.de
}

\begin{abstract}
The Compressed Baryonic Matter (CBM) experiment aims at exploring the QCD phase diagram at large baryon densities in the beam kinetic energy range from $2 \mathrm{~A} \mathrm{GeV}$ to $11(40) \mathrm{AGeV}$ (corresponding to center-of-mass energies of $2.7<\sqrt{s_{\mathrm{NN}}}<4.9(8.9) \mathrm{GeV}$ ) at the SIS-100 (SIS-300) accelerator of FAIR/GSI. The CBM detector is designed to measure in a triggerless readout mode both bulk observables with large acceptance and rare diagnostic probes such as charmed particles and vector mesons decaying into lepton pairs with an unprecedented interaction rate of up to $10 \mathrm{MHz}$. This contribution describes the concept of the experiment and its overall physics program and highlights observables that are sensitive to the phase structure like multi-strange hyperons.
\end{abstract}

11th International Workshop on Critical Point and Onset of Deconfinement - CPOD2017

7-11 August, 2017

The Wang Center, Stony Brook University, Stony Brook, NY

${ }^{*}$ Speaker. 


\section{Motivation}

Studying the strong forces between the constituents of nuclear matter-quarks, antiquarks, and gluons - under standard conditions for temperature and pressure e.g. by gamma-ray spectroscopy of radionuclides is restricted to the interaction of composite objects, protons and neutrons, which are three-quark bound states that behave like a liquid and serve as building blocks for all elements in the universe. In fact, approaching experimentally only hadronic states of strongly interacting matter would block the view of the actual variety of quark and gluon interactions that quantum chromodynamics (QCD) allows for depending —in a thermodynamic picture —on the temperature and the density of the system under study. In astrophysical objects like neutron stars [1] resulting from the core collapse of a Type II supernova nuclear matter is constrained by density conditions which significantly differ from those on the Earth's surface. On the high temperature side, looking back in time to beyond Big Bang baryogenesis [2] a plasma of free quarks and gluons is assumed to have existed for a few milliseconds. Such extreme states of nuclear matter are accessible by theory only for vanishing baryon densities/baryon chemical potentials $\mu_{\mathrm{B}}$ [3]. For larger densities, input from experimental findings is required to extrapolate from the point where first-principle QCD calculations are not applicable anymore. By means of colliding two heavy ions at (ultra)relativistic energies the hadronic phase (cf. Fig. 1) of strongly interacting matter can be overcome for a fraction of a second $\left(\sim 10^{-22} \mathrm{~s}\right)$ before the system hadronizes again.

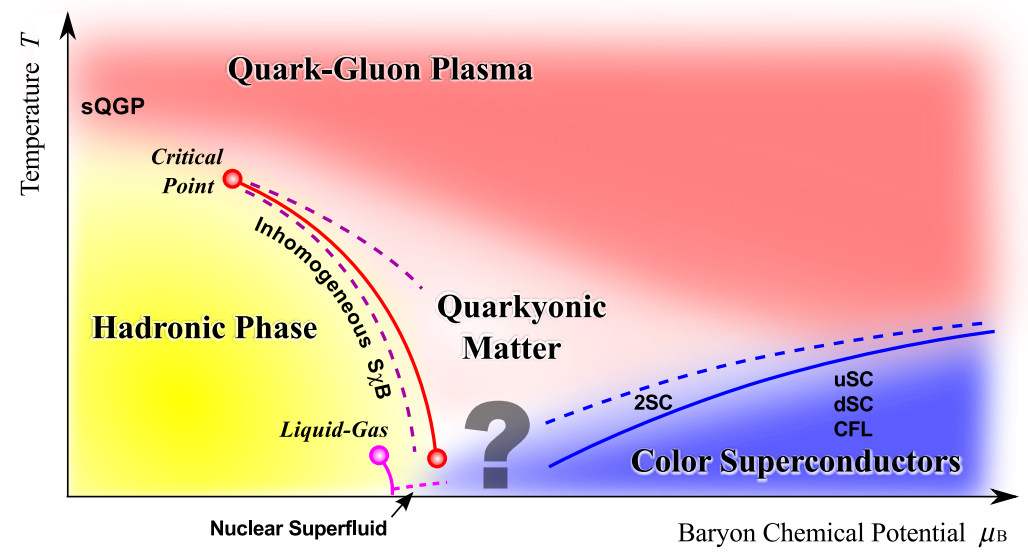

Figure 1: Possible phases and transitions of strongly interacting matter currently under theoretical and experimental investigation. At low temperature $T$ and low baryon chemical potential $\mu_{\mathrm{B}}$ quarks and antiquarks exist in confined and chirally asymmetric hadronic states. By increasing unilaterally the temperature at low $\mu_{\mathrm{B}}$ a cross-over to a deconfined and chirally symmetric phase of free quarks and gluons is expected [4]. At intermediate baryon chemical potential the hadronic phase is supposed to be separated from quarkyonic matter by a first-order phase transition which corresponds to the restoration of chiral symmetry while preserving confinement [5]. The critical endpoint towards low $T$ of this transition, in particular, is subject to present-day and future experimental nuclear research. Out of scope for research activities in the laboratory, at least according to the current state of knowledge, is the anticipated onset of color superconductivity at low $T$ and high $\mu_{\mathrm{B}}[6]$. Figure adapted from [3].

Depending on collision parameters different regions of the QCD phase diagram can be explored. When in the ALICE experiment [7] at the Large Hadron Collider (LHC) at CERN lead nuclei 
are collided at center-of-mass energies of $\sqrt{s_{\mathrm{NN}}}=2.76 \mathrm{TeV}$ the evolution of the collision system passes through the cross-over between the hadronic and the quark-gluon phase at high $T$ and low $\mu_{\mathrm{B}}$. A similar scenario is studied by the STAR experiment [8] at the Relativistic Heavy Ion Collider (RHIC) at Brookhaven National Laboratory where energies of up to $200 \mathrm{GeV}$ are obtained in gold-on-gold collisions. If the critical endpoint which is predicted at non-vanishing $\mu_{\mathrm{B}}$ and the firstorder phase transition between hadronic and quarkyonic matter (cf. Fig. 1) are accessible at these ultra-relativistic collision energies, yet needs to be proven. The heavy-ion program at the future Facility for Antiproton and Ion Research (FAIR) [9], in contrast, is especially designed for tracking down the possible impact of phase-boundary effects on the collision fireball evolution in the aforementioned region of intermediate to high baryon chemical potentials. Gold-on-gold collisions in the energy range of $\sqrt{s_{\mathrm{NN}}}=2.7-4.9 \mathrm{GeV}$ available at the SIS-100 accelerator-possibly extended to $8.9 \mathrm{GeV}$ with a SIS-300 upgrade - will be recorded by the Compressed Baryonic Matter (CBM) experiment [10]. The operation of the CBM spectrometer with SIS-100 heavy-ion beams is absolutely unique considering the design beam-target interaction rate of $10 \mathrm{MHz}$ throughout the entire energy range covered (cf. Fig. 2). Any other experiment capable of running at SIS-100 energies would require at least a factor of 100 more beam-on-target time than CBM to accumulate the same amount of statistics for an observable under study.

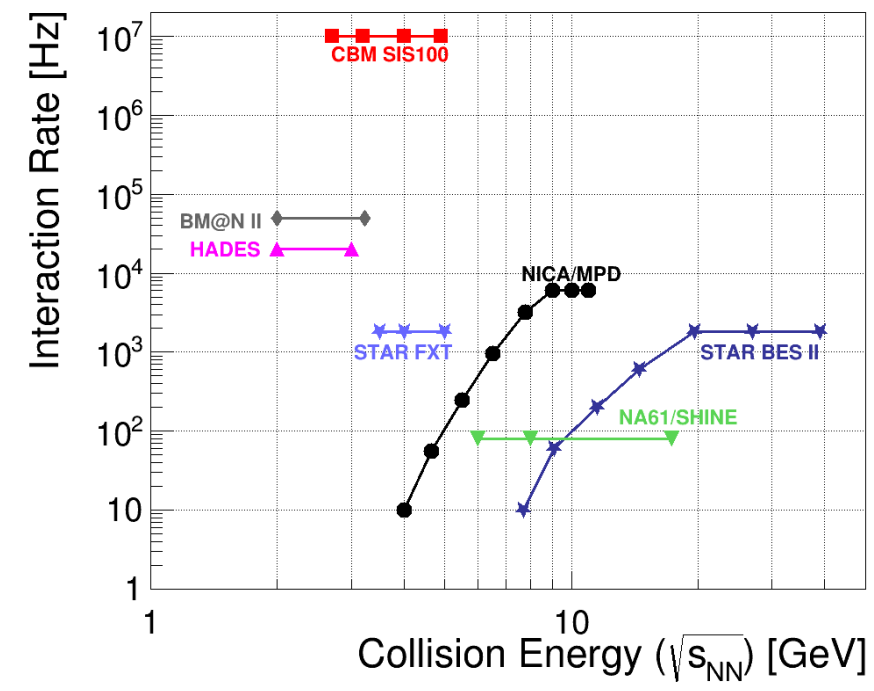

Figure 2: Comparison of available interaction rates at different heavy-ion experiments as a function of collision energy $\sqrt{s_{\mathrm{NN}}}$. Experiments with invariant interaction rates (HADES, BM@N II, CBM SIS100, STAR FXT, and NA61/SHINE) are designed to operate with a fixed target, NICA/MPD and STAR BES II work in collider mode. Figure taken from [11].

The demand for high statistics arises from the sensitivity of rare reaction probes to critical phenomena and the role of additional partonic degrees of freedom in the vicinity of a phase transition during the fireball evolution. Section 2 introduces the most relevant of these probes whose accurate detection determines the entire design of the CBM apparatus. The subsystems of CBM, their dedicated purposes, and the novel free-streaming readout paradigm of the experiment which is a prerequisite to measure at such unprecedented interaction rates are sketched in Sec. 3. A brief 
outlook summarizing the major activities of the collaboration before CBM goes into operation in 2024 concludes this contribution in Sec. 4.

\section{Multi-strange rare probes at SIS-100 energies}

The physics program [11] of the CBM experiment in the SIS-100 energy range comprises both bulk observables and rare probes. Among the former are the collective flow of hadrons and particle correlations like event-by-event fluctuations of conserved quantities. The latter are dilepton pairs $\left(e^{+} e^{-}\right.$and $\mu^{+} \mu^{-}$), particles carrying charm especially when running close to or below the kinetic production threshold, either openly ( $D$ mesons) or hiddenly $(J / \psi$ mesons), and multi-strange hyperons, both individually and bound e.g. in hypernuclei.

Due to the relatively small bare mass of strange quarks $\left(m_{s} \sim 100 \mathrm{MeV} / \mathrm{c}^{2}\right)$ compared to the nextin-weight charm quarks $\left(m_{c} \sim 1.29 \mathrm{GeV} / \mathrm{c}^{2}\right)$ strange mesons and baryons are kinetically produced already at low $\sqrt{s_{\mathrm{NN}}}$. In this energy range which is covered by the SIS-100 accelerator, a particularly dense collision zone is expected to be formed. As nucleons which participate in a heavy-ion reaction do not carry any strangeness, the $s$ and $\bar{s}$ quarks observed in reaction products must be created during the collision. Thus, strange mesons and baryons can serve as probes for the conditions in the dense fireball. The properties of the nuclear medium and the hadronic and partonic influences therein actually impact strangeness production to a significant extent. Data from the HADES experiment on sub-threshold production of $\Xi^{-}(d s s)$ baryons in $\mathrm{Ar}+\mathrm{KCl}$ collisions at SIS-18 energies $\left(\sqrt{s_{\mathrm{NN}}}=2.61 \mathrm{GeV}\right)$ [12] show a yield per event which is enhanced by a factor of about 20 compared to the expectation from a statistical model fit to all particle yields. This finding indicates different chemical freeze-out conditions for strange hyperons at sub-threshold energies as compared to bulk hadrons. While theoretical progress has been made in attributing the kink-shaped enhancement of the $K^{+} / \pi^{+}$yield ratio measured by the NA49 collaboration [13] to a partial restoration of chiral symmetry in the collision zone (cf. Fig. 3) similar conclusions cannot be drawn from a beam-energy scan of $\Xi^{-}$yields due to a lack of data in the SIS-100 energy range.

Considering the (sub-threshold) production of $\Omega$ (sss) and anti- $\Omega(\bar{s} \bar{s} \bar{s})$ baryons no statistically relevant experimental data on production yields exist at all below $\sqrt{s_{\mathrm{NN}}}=8.9 \mathrm{GeV}$ [17]. A systematic high-precision $\Omega$ measurement would shed more light on the impact of deconfinement and chiral symmetry restoration (CSR) during the fireball evolution on strangeness production. PHSD transport model calculations of $\bar{\Omega}^{+}$and $\Omega^{-}$multiplicities (cf. Fig. 4) indeed suggest a strong $\bar{\Omega}^{+}$ enhancement in particular at SIS-100 energies if partonic degrees of freedom are included.

A rough estimate- based on the PHSD results—of $\Omega$ production yields per day of continuous CBM operation at $4.7 \mathrm{GeV}$ center-of-mass energy gives accumulated $3.7 \times 10^{6} \bar{\Omega}^{+}$and $1.1 \times 10^{7}$ $\Omega^{-}$baryons within 24 hours of data taking. Here, a sustained (integrating over accelerator duty cycles) beam-target interaction rate of $10 \mathrm{MHz}$, a conversion factor of 0.1 from the simulated central collisions to minimum bias cross section, and detection efficiencies (acceptance and reconstruction) of $2.3 \%$ for $\bar{\Omega}^{+}$and $4.3 \%$ for $\Omega^{-}$are assumed according to recent CBM physics simulations [19]. These numbers allow for pioneering systematic studies of multi-strange baryons and can be used to constrain transport model predictions of strangeness enhancement to the level that an impact of 

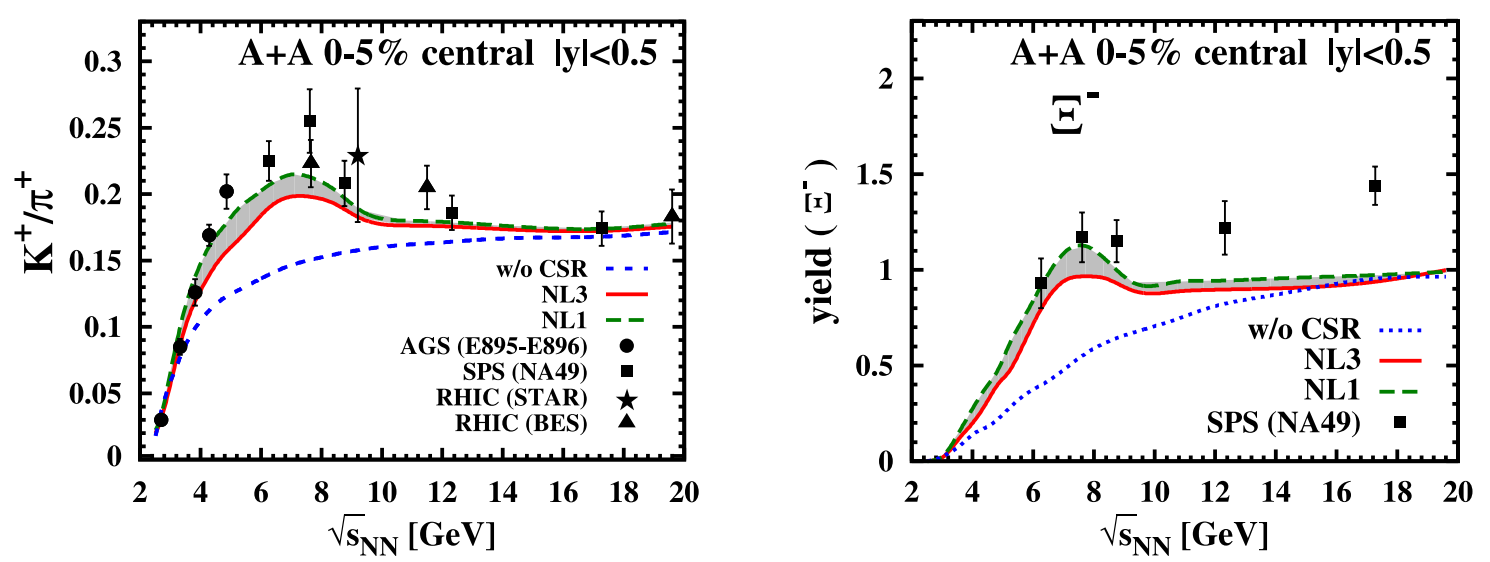

Figure 3: Impact of chiral symmetry restoration (CSR) on the descriptive power of the PHSD transport model $[14,15]$ regarding the measured excess in the $K^{+} / \pi^{+}$yield ratio at $\sqrt{s_{\mathrm{NN}}}<10 \mathrm{GeV}$ (left plot). The effect of activating CSR in the model on the $\Xi^{-}$yield (right plot) cannot be constrained quantitatively due to the unavailability of data in the SIS-100 energy range. The red and green curve differ in the applied nuclear compression modulus resulting in a "stiff" nuclear equation of state (EOS) in the former and a "soft" EOS in the latter case. Figures taken from [16].
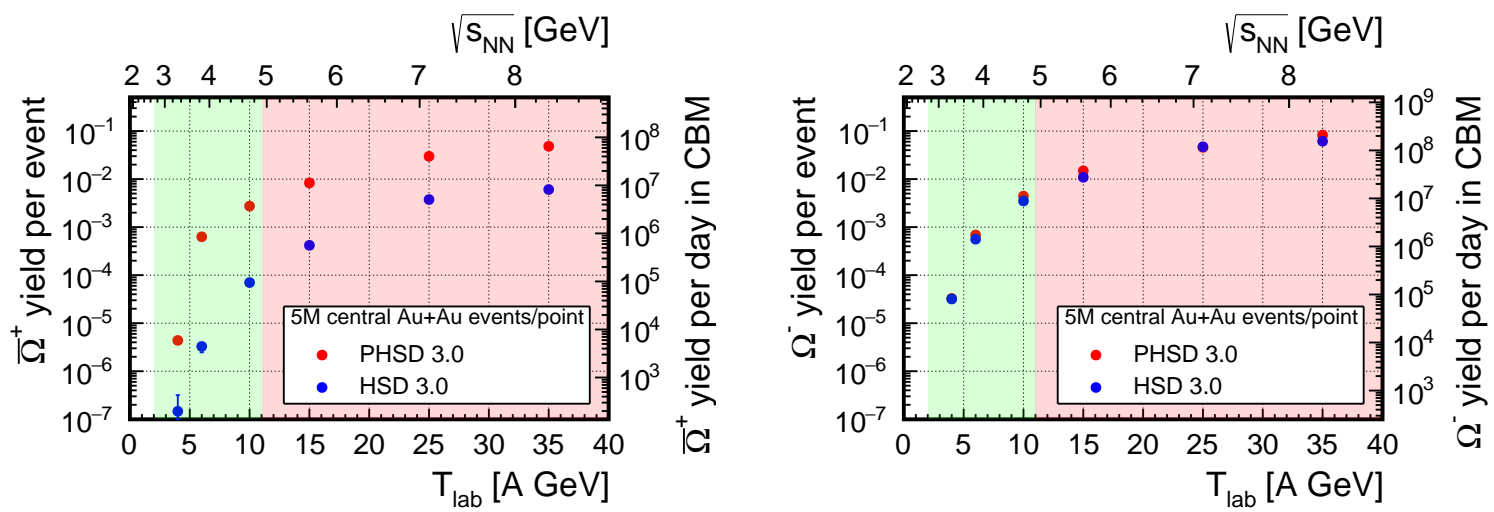

Figure 4: Beam energy dependence of $\bar{\Omega}^{+}$(left plot) and $\Omega^{-}$(right plot) baryon multiplicities (yield per event) as computed with the (P)HSD 3.0 code. Partonic degrees of freedom in the transport model lead to a significant excess yield for $\bar{\Omega}^{+}$baryons, notably in the green shaded SIS-100 energy range. The right-hand ordinates give an estimate of the production yield expected at sustained $10 \mathrm{MHz}$ gold-on-gold interaction rates with minimum collision centrality bias in the CBM experiment. Multiplicities taken from [18].

phases other than the hadronic one on production yields can either be quantitatively confirmed or excluded.

\section{The CBM experiment}

Recording primary and secondary reaction products of up to $10^{7}$ heavy-ion collisions per second necessitates - apart from fast responding and radiation hard detectors - a data acquisition strategy that is not restricted by any trigger and readout latency on the order of (at least) several $\mu \mathrm{s}$ as introduced by a typical centrally controlled trigger hierarchy. All front-end electronics in CBM 
therefore digitize and timestamp the analog detector response and transmit digital messages without an explicit trigger request, i.e. in a self-triggered manner, towards a high-performance computing farm [20]. On and across dedicated compute nodes, event reconstruction and selection software processes a continuous stream of incoming data which are sorted by time and source, i.e. subsystem, but yet need to be partitioned into physical events with sophisticated and efficient algorithms [21]. In fact, the decay topology of rare probes measured by CBM does not create a distinctive response signature in the detector that could be utilized for low-level hardware triggering. These two key aspects-the need to accumulate sufficient statistics for rare-probe studies and the necessity of a high-level software trigger to spot interesting event candidates-are constitutive of the free-streaming readout paradigm of CBM.

During the (largely completed) R\&D phase of the different detector subsystems (cf. Fig. 5) high diligence was devoted to the compliance of all components with the DAQ paradigm. The cart horse

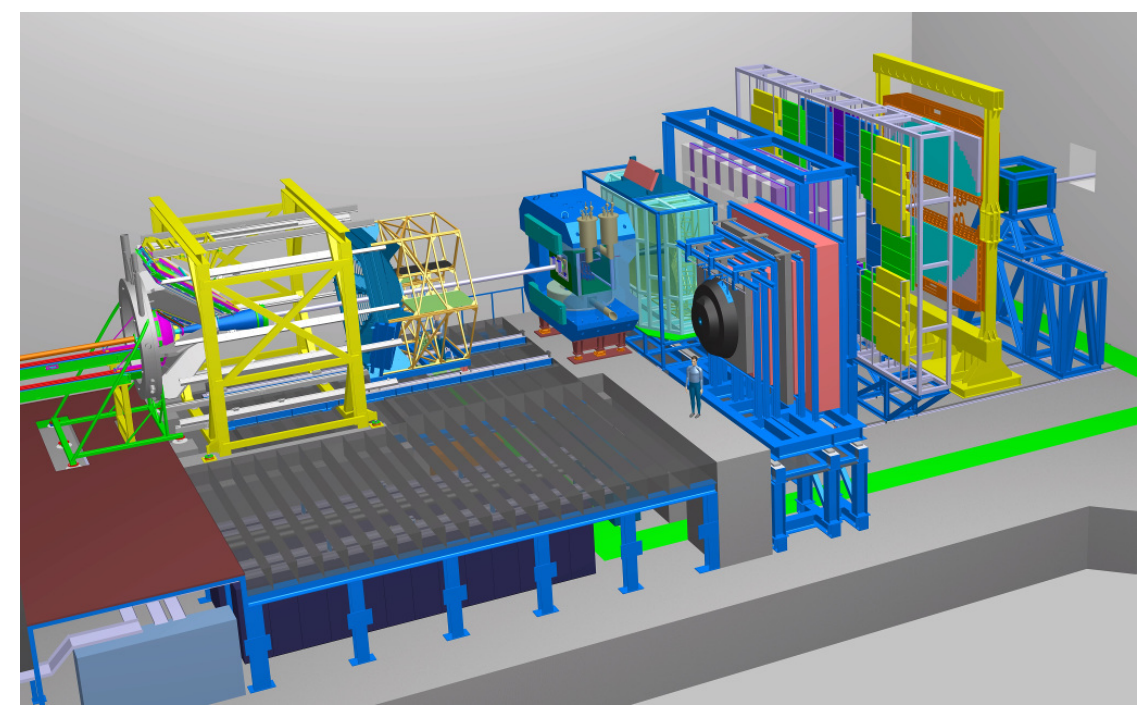

Figure 5: Virtual view into the CBM cave depicting the detached tandem setup of the HADES and CBM spectrometers. Focusing on CBM from left to right following the beam direction, the setup starts with a superconducting dipole magnet which is positioned on the concrete block in the figure center. Its gap houses a vacuum vessel containing the target and a micro-vertex detector (MVD) behind which a silicon tracking system (STS) is located. The next subsystem in line is a ring-imaging Cherenkov (RICH) detector which can —depending on the physics observable under study—be replaced by muon chambers (MuCh). Further towards the beam dump, a transition radiation detector (TRD) is followed by a time-of-flight (TOF) wall, an electromagnetic calorimeter (ECAL), and a projectile spectator detector (PSD).

of (online) particle identification (PID) in CBM is its Silicon Tracking System (STS) [22] which resides in a magnetic dipole field with a bending power of $1 \mathrm{Tm}$ [23] allowing for momenta of charged particle tracks to be determined with $\Delta p / p<1 \%$. From the geometrical track information of hyperon daughter particles (e.g. $\bar{\Omega}^{+} \rightarrow \bar{\Lambda} K^{+}$or $\Omega^{-} \rightarrow \Lambda K^{-}$with subsequent $\bar{\Lambda} \rightarrow p^{-} \pi^{+}$or $\Lambda \rightarrow p \pi^{-}$ decays) the decay vertex of the mother particle can be determined in the STS without identifying the individual daughter particle species. For event selection algorithms running online, this is a very beneficial scenario as combining data from different subsystems results in additional computational 
costs. It might, however, be necessary to reduce the combinatorial background of invariant-mass spectra if the signal-over-background ratio otherwise became critical (as for $\bar{\Omega}^{+}$baryons and for double- $\Lambda$ hypernuclei). In this case, the PID backbone of CBM-its large-area Time-of-Flight (TOF) wall composed of multi-gap resistive plate chambers (MRPC) [24]—provides full particle identification by supplementing the track momentum measured by the STS with velocity information. The TOF subsystem is designed to determine the time of flight of bulk hadrons, i.e. pions, kaons, protons, and their respective antiparticles, with a precision of $80 \mathrm{ps}$.

As decay vertices of $D$ mesons ( $c \tau=311.8 \mu \mathrm{m}$ for $D^{+/-}$at $\gamma=1$ in the laboratory) carrying open charm are located much closer to the collision vertex than the decay vertices of hyperons ( $c \tau=7.89 \mathrm{~cm}$ for $\Lambda, c \tau=2.46 \mathrm{~cm}$ for $\Omega$ ) additional tracking layers in front of the STS in close vicinity to the target form the Micro-Vertex Detector (MVD) [25] dedicated to open-charm studies. Particle identification in the dilepton program of CBM is primarily carried out by the Ring-Imaging Cherenkov (RICH) detector [26] and - complementarily - the Muon Chambers (MuCh) [27], supported by the Transition Radiation Detector (TRD) [28] and the Electromagnetic Calorimeter (ECAL) [29]. Collision centrality and reaction plane determination is done by the Projectile Spectator Detector (PSD) [30].

\section{Outlook}

The CBM experiment being designed for taking data at the SIS-100 synchrotron of the FAIR facility is expected to take first data in 2024. With the R\&D phase of detector development almost finished and in order to prepare for a short commissioning time the CBM project enters into a phase where subsystems are used for contributions to the physics program in existing experiments or where CBM system aspects are being addressed (FAIR phase 0). The former comprises installing the MAPMT photosensors of the CBM RICH detector as readout elements of the HADES RICH, installing CBM-TOF MRPC counter modules as endcap TOF detection system in the STAR experiment at RHIC [31], and using the PSD and STS detectors at the BM@N experiment in JINR. The latter is the main focus of the "mini"-CBM (mCBM) experiment [32] that is currently being constructed in the experimental hall of the SIS-18 accelerator at GSI/FAIR. Here, a slice of CBM with all detector components, i.e. MVD, STS, MUCH, RICH, TRD, TOF, ECAL, and PSD, will be installed to demonstrate the capability to run at an interaction rate of $10 \mathrm{MHz}$ of $\mathrm{Au}+\mathrm{Au}$ reactions. The data will be transferred by CBM data acquisition components into the Green IT Cube of GSI/FAIR where dedicated algorithms will inspect the incoming data stream for the reconstruction of $\Lambda$-hyperons that are produced in the SIS-18 energy range close to or below their production threshold in elementary nucleon-nucleon collisions. The mCBM experiment will start taking data in 2018 .

\section{Acknowledgments}

The project is partially funded by BMBF grant no. 05P15VHFC1 and by the FuturePID work package (WP19) of the HadronPhysics3 activity within the EU's Seventh Framework Programme (FP7). 


\section{References}

[1] J. M. Lattimer and M. Prakash, The Physics of Neutron Stars, Science 304 (2004) 536, [astro-ph/0405262].

[2] I. Affleck and M. Dine, A new mechanism for baryogenesis, Nucl. Phys. B 249 (1985) 361.

[3] K. Fukushima and T. Hatsuda, The phase diagram of dense QCD, Rep. Prog. Phys. 74 (2011) 014001, [1005.4814].

[4] D. H. Rischke, The quark-gluon plasma in equilibrium, Prog. Part. Nucl. Phys. 52 (2004) 197, [nucl-th/0305030].

[5] L. McLerran and R. D. Pisarski, Phases of dense quarks at large $N_{c}$, Nucl. Phys. A 796 (2007) 83, [0706.2191].

[6] M. G. Alford, A. Schmitt, K. Rajagopal and T. Schäfer, Color superconductivity in dense quark matter, Rev. Mod. Phys. 80 (2008) 1455, [0 709 . 4635].

[7] ALICE collaboration, K. Aamodt et al., The ALICE experiment at the CERN LHC, J. Instrum. 3 (2008) S08002.

[8] STAR collaboration, J. Adams et al., Experimental and theoretical challenges in the search for the quark-gluon plasma: The STAR Collaboration's critical assessment of the evidence from RHIC collisions, Nucl. Phys. A 757 (2005) 102, [nucl-ex/0501009].

[9] H. Gutbrod et al., eds., FAIR Baseline Technical Report. GSI, Darmstadt, Germany, 2006.

[10] CBM Collaboration, Compressed Baryonic Matter Experiment - Technical Status Report. GSI, Darmstadt, Germany, 2005.

[11] CBM collaboration, T. Ablyazimov et al., Challenges in QCD matter physics - The scientific programme of the Compressed Baryonic Matter experiment at FAIR, Eur. Phys. J. A 53 (2017) 60, [1607.01487].

[12] HADES collaboration, G. Agakishiev et al., Statistical hadronization model analysis of hadron yields in $p+N b$ and Ar + KCl at SIS18 energies, Eur. Phys. J. A 52 (2016) 178, [1512.07070].

[13] NA49 collaboration, C. Alt et al., Pion and kaon production in central Pb + Pb collisions at 20A and 30A GeV: Evidence for the onset of deconfinement, Phys. Rev. C 77 (2008) 024903, [0710 . 0118].

[14] W. Cassing and E. L. Bratkovskaya, Parton transport and hadronization from the dynamical quasiparticle point of view, Phys. Rev. C 78 (2008) 034919, [0 808 . 0022 ].

[15] W. Cassing and E. L. Bratkovskaya, Parton-hadron-string dynamics: An off-shell transport approach for relativistic energies, Nucl. Phys. A 831 (2009) 215, [0907.5331].

[16] A. Palmese, W. Cassing, E. Seifert, T. Steinert, P. Moreau and E. L. Bratkovskaya, Chiral symmetry restoration in heavy-ion collisions at intermediate energies, Phys. Rev. C 94 (2016) 044912, [1607.04073].

[17] C. Blume, Energy dependence of hadronic observables, J. Phys. G: Nucl. Part. Phys. 31 (2005) S57.

[18] CBM collaboration, V. Friese, Strangeness Prospects with the CBM Experiment, J. Phys.: Conf. Ser. 668 (2016) 012014.

[19] I. Vassiliev. private communication, 2017.

[20] CBM collaboration, J. de Cuveland and V. Lindenstruth, A First-Level Event Selector for the CBM Experiment at FAIR, J. Phys.: Conf. Ser. 331 (2011) 022006. 
[21] I. Kisel, Scientific and high-performance computing at FAIR, Eur. Phys. J. Web Conf. 95 (2015) 01007.

[22] CBM collaboration, J. Heuser et al., eds., Technical Design Report for the CBM Silicon Tracking System (STS). GSI, Darmstadt, 2013.

[23] CBM collaboration, A. Malakhov and A. Shabunov, eds., Technical Design Report for the CBM Superconducting Dipole Magnet. GSI, Darmstadt, 2013.

[24] CBM collaboration, N. Herrmann, ed., Technical Design Report for the CBM Time-of-Flight System (TOF). GSI, Darmstadt, 2014.

[25] M. Koziel et al., Vacuum-compatible, ultra-low material budget Micro-Vertex Detector of the compressed baryonic matter experiment at FAIR, Nucl. Instrum. Meth. A 845 (2017) 110.

[26] CBM collaboration, C. Höhne, ed., Technical Design Report for the CBM Ring Imaging Cherenkov (RICH) Detector. GSI, Darmstadt, 2013.

[27] CBM collaboration, S. Chattopadhyay, Y. P. Viyogi, P. Senger, W. F. J. Müller and C. J. Schmidt, eds., Technical Design Report for the CBM Muon Chambers (MuCh). GSI, Darmstadt, 2015.

[28] M. Petriş et al., TRD detector development for the CBM experiment, Nucl. Instrum. Meth. A 732 (2013) 375

[29] I. E. Korolko, M. S. Prokudin and Y. M. Zaitsev, The CBM ECAL, J. Phys.: Conf. Ser. 798 (2017) 012164.

[30] CBM collaboration, F. Guber and I. Selyuzhenkov, eds., Technical Design Report for the CBM Projectile Spectator Detector (PSD). GSI, Darmstadt, 2015.

[31] STAR collaboration and CBM collaboration eTOF Group, Physics Program for the STAR/CBM eTOF Upgrade, 1609.05102.

[32] CBM collaboration, mCBM@SIS18-A CBM full system test-setup for high-rate nucleus nucleus collisions at GSI / FAIR. GSI, Darmstadt, 2017. 